\title{
SURFACE WATER WAVES DUE TO AN OSCILLATORY WAVEMAKER IN THE PRESENCE OF SURFACE TENSION
}

\author{
B.N. MANDAL \\ Physical and Earth Sciences Division \\ Indian Statistical Institute \\ 203, B.T. Road \\ Calcutta - 700035 India \\ and \\ S. BANERJEA \\ Calcutta Mathematical Society \\ 92 , A.P.C. Road \\ Calcutta - 700009 India
}

(Received March 28, 1990)

\begin{abstract}
The initial value problem of generation of surface water waves by a harmonically oscillating plane vertical wavemaker in an infinite incompressible fluid under the action of gravity and surface tension is investigated. In the asymptotic evaluation of the free surface depression for large time and distance, the contribution to the integral by stationary phase method gives rise to transient component of the free surface depression while the contribution from the poles give rise to steady state component. It is observed that the presence of surface tension sometimes changes the qualitative nature of the transient component of free surface depression.
\end{abstract}

KEY WORDS AND PHRASES. Water waves, gravity waves and gravity-capillary waves. 1991 AMS SUBJECT CLASSIFICATION CODE. 76N15, 76C.

1. INTRODUCTION. The forced waves produced by a time harmonic plane vertical wavemaker was treated by Havelock [1] long back within the framework of linearized theory of water waves assuming the irrotational motion of the liquid. Later Rhodes-Robinson [2] extended the same problem to include the effect of surface tension and obtained the solution of the boundary value problem using a method based on the application of Green's integral theorem. Recently Faltas [3] solved the initial value problem of generation of surface waves by harmonically oscillating vertical wavemaker using the generalized function method and also presented the asymptotic behavior of free surface depression for large time and distance.

The present paper is an extension of the problem considered in [3] to include the effect of surface tension at the free surface. An asymptotic analysis of the free surface depression for large time and distance is presented.

\section{FORMULATION OF THE PROBLEM.}

We are concerned with the transient development of the two dimensional surface water waves generated by a harmonically oscillating plane vertical wavemaker in the presence of surface tension at the free surface. We use a rectangular Cartesian coordinate system in which the origin is taken at the 'edge' where the wavemaker meets the free surface and $y$-axis is taken vertically downwards 
so that $y=0, x>0$ is the undisturbed free surface and $x=0$ is the wavemaker. In the undisturbed state the fluid occupies the region $x \geq 0$ and $0 \leq y \leq h, h$ being the depth of the fluid which is assumed to inviscid and incompressible. The motion in the fluid is generated by forced harmonic oscillation of the wavemaker along the horizontal direction which is switched on at $t=0$ so that the horizontal velocity of the wavemaker is given by

$$
U(y, t)=U_{1}(y) e^{-i \sigma t} H(t)
$$

where $u_{1}(y)$ is an arbitrary function of $y, \sigma$ is the frequency and $H(t)$ is the Heaviside function. Since the motion starts from rest, it is irrotational and can be described by a potential function $\varphi(x, y, t)$ which satisfies the following initial value problem described by

$$
\nabla^{2} \varphi=0 \quad \text { in } 0 \leq x<\infty, \quad 0 \leq y \leq h, t>0,
$$

with the bottom condition

$$
\frac{\partial \varphi}{\partial y}=0 \quad \text { on } y=h, t>0,
$$

The linearized dynamic and kinematic conditions are

$$
\left.\begin{array}{c}
\frac{\partial \varphi}{\partial y}=\frac{\partial \eta}{\partial t}, \\
\rho g \eta-\rho \varphi_{t}-T \eta_{x x}=0
\end{array}\right\} \text { on } y=0, t>0
$$

where $\eta \equiv \eta(x, t)$ is the free surface depression, $g$ is the gravity and $T$ is the co-efficient of surface tension, the wavemaker condition

$$
\frac{\partial \varphi}{\partial x}=U(y, t)=U_{1}(y) e^{-i \sigma t} H(t) \quad \text { on } x=0, t>0,
$$

the initial conditions

$$
\varphi=\eta=0 \text { at } t=0,
$$

and also, the edge condition prescribing the free surface slope at the wavemaker as

$$
\varphi_{x y}=\lambda(t)=\lambda_{1} e^{-i \sigma t} H(t)
$$

where $\lambda_{1}$ is a known constant. Note that $\varphi_{x y}$ is discontinuous at the edge since

$$
\varphi_{x y}(o, o+, t)=U_{1}^{\prime}(o) e^{-i \sigma t} H(t)
$$

so that $\varphi(x, y, t)$ is weakly singular at the edge (cf. Rhodes-Robinson [2]).

Here we assume that $\varphi$ and $\eta$ are generalized functions of $x$ in the sense of Lighthill [5] so that their Fourier cosine transforms exist with respect to $x$.

\section{SOLUTION OF THE PROBLEM.}

Introducing the Fourier cosine transform with respect to $x$ and Laplace transform with respect to $t$ as

$$
\bar{F}_{c}(k, y, s)=\sqrt{\frac{2}{\pi}} \int_{0}^{\infty} \operatorname{coskx}\left\{\int_{0}^{\infty} e^{-s t} F(x, y, t) d t\right\} d x
$$

where the subscript $c$ and bar refer to the Fourier cosine transform respectively, (2.1) to (2.6) give

$$
\begin{gathered}
\frac{\partial^{2}}{\partial y^{2}} \bar{\varphi}_{c}-k^{2} \bar{\varphi}_{c}=\sqrt{\frac{2}{\pi}} \bar{U}, \\
\frac{\partial}{\partial y} \bar{\varphi}_{c}=0 \text { on } y=h, s>0,
\end{gathered}
$$




$$
\left.\begin{array}{c}
\frac{\partial}{\partial y} \bar{\varphi}_{c}=s \bar{\eta}_{c}, \\
\bar{\varphi}_{c}=\frac{g}{s}(1+M k) \bar{\eta}_{c}+\frac{2}{\pi} \frac{M \bar{\lambda} g}{s^{2}}
\end{array}\right\} \text { on } y=0, s>0
$$

where $M=T / \rho g$. Solutions for $\bar{\eta}_{c}$ and $\bar{\varphi}_{c}$ are given by

$$
\begin{gathered}
\sqrt{\frac{\pi}{2}} \bar{\eta}_{c}=-\frac{M \bar{\lambda}}{1+M k^{2}}\left(\frac{1}{s}-\frac{s}{\alpha^{2}+s^{2}}\right)-\frac{s}{\alpha^{2}+s^{2}} \int_{o}^{h} \frac{\operatorname{coshk}(h-\xi)}{\operatorname{coshkh}} \bar{U}(\xi, s) d \xi \\
\sqrt{\frac{\pi}{2}} \bar{\varphi}_{c}=\int_{o}^{y} k^{-1} \operatorname{sinhk}(y-\xi) \bar{U}(\xi, x) d \xi+\frac{M \bar{\lambda} g \operatorname{coshk}(h-y)}{\left(\alpha^{2}+s^{2}\right) \operatorname{coshkh}} \\
-\frac{g\left(1+M k^{2}\right) \operatorname{coshky}}{\left(\alpha^{2}+s^{2}\right) \operatorname{coshkh}} \int_{o}^{h} \frac{\operatorname{coshk}(h-\xi)}{\operatorname{coshkh}} \bar{U}(\xi, s) d \xi \\
-\frac{\operatorname{sinhky}}{k} \int_{o}^{h} \frac{\operatorname{coshk}(h-\xi)}{\operatorname{coshkh}} \bar{U}(\xi, s) d \xi
\end{gathered}
$$

where

$$
\alpha^{2}=g k\left(1+M k^{2}\right) \operatorname{tanhkh} .
$$

The inverse Laplace and Fourier cosine transforms together with convolution theorem for Laplace transform employed to (3.4), (3.5) after using the form of $U(y, t)$ and $\lambda(t)$ from (2.4) and (2.6) respectively, give rise to

$$
\begin{aligned}
\frac{\pi}{2} \eta(x, t)= & \int_{0}^{\infty} \operatorname{coskx} \frac{M \lambda_{1}}{1+M k^{2}} \frac{i}{\sigma}\left(1-e^{-i \sigma t}\right) d k \\
& +\int_{0}^{\infty} \operatorname{coskx}\left(\frac{M \lambda_{1}}{1+M k^{2}}-\beta(k)\right)\left(\frac{\alpha \sin \alpha t+i \sigma \cos \alpha t-i \sigma e^{-i \sigma t}}{\alpha^{2}-\sigma^{2}}\right) d k \\
\frac{\pi}{2} \varphi(x, y, t)= & \int_{0}^{\infty} \operatorname{coskx} \frac{M \lambda_{1} g \operatorname{coshk}(h-y)}{\alpha \operatorname{coshkh}}\left(\frac{\alpha \cos \alpha t-\alpha e^{-i \sigma t}-i \sigma \sin \alpha t}{\alpha^{2}-\sigma^{2}}\right) \beta(k) d k \\
& -\int_{o}^{\infty} \operatorname{coskx} \frac{\operatorname{sinhky}}{k} e^{-i \sigma t} \beta(k)+\int_{o}^{\infty} \operatorname{coskx} \gamma(k, y) e^{-i \sigma t} d k
\end{aligned}
$$

where

and

$$
\beta(k)=\int_{0}^{h} \frac{U_{1}(\xi) \cosh (h-\xi)}{\operatorname{coshkh}} d \xi
$$

$$
\gamma(k, y)=\int_{o}^{y} k^{-1} \sinh \mathrm{k}(y-\xi) U_{1}(\xi) d \xi
$$

4. ASYMPTOTIC EVALUATION OF FREE SURFACE DEPRESSION.

We are interested to find the form of the free surface depression $\eta(x, t)$ for large values of $x$ and $t$. We note

$$
\eta=I+J
$$

where

$$
\begin{gathered}
I=\frac{2}{\pi} \int_{0}^{\infty} \frac{i}{\sigma} \frac{M \lambda_{1}}{1+M k^{2}} \operatorname{coskxdk}-\frac{2}{\pi} \int_{0}^{\infty} e^{-i \sigma t} i\left[\frac{M \lambda_{1}}{1+M k^{2}} \frac{\alpha^{2}}{\sigma\left(\alpha^{2}-\sigma^{2}\right)}-\frac{\sigma \beta(k)}{\alpha^{2}-\sigma^{2}}\right] d k \\
J=\frac{2}{\pi} \int_{0}^{\infty} \operatorname{coskx}\left[\frac{M \lambda_{1}}{1+M k^{2}}-\beta(k)\right]\left(\frac{\alpha \sin \alpha t+i \sigma \cos \alpha t}{\alpha^{2}-\sigma^{2}}\right) d k
\end{gathered}
$$


Now (4.1) and (4.2) can be rewritten as follows

where

$$
\begin{gathered}
I=\frac{2 i}{\pi} e^{-i \sigma t} \sum_{n=1}^{4} I_{n}+\frac{2 i}{\pi} I_{5}, \\
J=\frac{2}{\pi} \sum_{n=1}^{4} J_{n}
\end{gathered}
$$

$$
\begin{gathered}
I_{1,2}= \pm \frac{1}{4} \int_{0}^{\infty} \frac{\beta(k)}{\alpha \mp \sigma}\left(e^{i k x}+e^{-i k x}\right) d k \\
I_{3,4}=-\frac{1}{4} \int_{0}^{\infty} \frac{M \lambda_{1}}{1+M k^{2}} \frac{e^{i k x}+e^{-i k x}}{\alpha \pm \sigma} d k \\
I_{5}=\frac{i}{2 \sigma} M \lambda_{1} \int_{-\infty}^{\infty} \frac{e^{i k x}}{1+M k^{2}} d k \\
J_{1,2}=-\frac{i}{4} \int_{0}^{\infty}\left[\frac{M \lambda_{1}}{1+M k^{2}}-\beta(k)\right] \frac{e^{i(\alpha t \pm k x)}}{\alpha+\sigma} d k \\
J_{3,4}=\frac{i}{4} \int_{0}^{\infty}\left[\frac{M \lambda_{1}}{1+M k^{2}}-\beta(k)\right] \frac{e^{i(\alpha t \pm k x)}}{\alpha-\sigma} d k
\end{gathered}
$$

The main contribution to the asymptotic values of the above integrals for large $x$ and $t$ comes from the poles and stationary points of the integrands. The contributions to the integrals from poles represent steady state component while those from stationary points represent transient component. Now the integrals, $I_{1}, I_{4}, J_{3}, J_{4}$ contain a pole at $k=k_{o}$ where $k_{o}$ is the unique positive real roof of the equation

$$
\left\{g k\left(1+M k^{2}\right) \operatorname{tanhkh}\right\}^{1 / 2}=\sigma
$$

Again the integrals $J_{2}, J_{4}$ contain the stationary points which are real positive zeros of the equation

$$
\alpha^{\prime}(k)=\frac{x}{t} .
$$

Now $\alpha^{\prime}(0)=(g h)^{1 / 2}$ while $\alpha^{\prime}(k) \rightarrow+\infty$ as $k \rightarrow \infty$, also $\alpha^{\prime}(k)$ is positive for large $k$. Hence $\alpha^{\prime}(k)$ has a finite number of local maxima and minima in the range $[0, \infty)$. In particular, for $M / h^{2}=.075$ it is observed that $\alpha^{\prime}(k)$ has one maximum and one minimum in $[0, \infty)$. Now if the value of $x / t$ is less than the global minimum of $\alpha^{\prime}(k)$ then there is no root of $\alpha^{\prime}(k)=\frac{x}{t}$ in the range $[0, \infty)$ so that there does not exist any transient part of free surface depression whereas for $x / t$ greater than the global minimum of $\alpha^{\prime}(k)$ there exists a finite number of stationary points. It may be noted that in the absence of surface tension, $\alpha^{\prime}(0)=(g h)^{1 / 2}, \alpha^{\prime}(k) \rightarrow 0$ as $k \rightarrow \infty$ and $\alpha^{\prime \prime}(k)>0$ for $k>0$, so that $\alpha^{\prime}(k)=x / t$ has a unique positive root if $x / t<(g h)^{1 / 2}$.

Now $I_{5}$ can be evaluated as

$$
I_{5}=\frac{i \pi}{2 \sigma} M^{1 / 2} \lambda_{1} e^{-M^{-1 / 2}}
$$

so that

$$
I_{5} \rightarrow 0 \quad \text { as } x \rightarrow \infty \text {. }
$$

The contributions due to the poles of the integrals $I_{1}, I_{4}, J_{3}, J_{4}$ can be evaluated as given in $[3,4]$ for large $x$ and $t$ as,

$$
I_{\text {polar }} \sim\left\{M E-\beta\left(k_{o}\right)\right\} \frac{e^{i k_{o} x}-e^{-i k_{o} x}}{2 \alpha^{\prime}\left(k_{o}\right)} e^{-i \sigma t}
$$




$$
J_{\text {polar }} \sim\left\{M E-\beta\left(k_{o}\right)\right\} \frac{e^{i k_{o} x}+e^{-i k_{o} x}}{2 \alpha^{\prime}\left(k_{o}\right)} e^{-i \sigma t}
$$

where $E=\frac{\lambda_{1}}{1+M k_{o}^{2}}$ and $\alpha^{\prime}\left(k_{o}\right)$ is the derivative of $\alpha$ at $k=k_{o}$.

Employing the stationary phase method we calculate the transient part of $J$ as

$$
\begin{aligned}
J_{t r} & \sim \sum_{j} i \sqrt{\frac{1}{2 \pi t \alpha^{\prime \prime}\left(k_{j}\right)}}\left\{\frac{M \lambda_{1}}{1+M k_{j}^{2}}-\beta\left(k_{j}\right)\right\} \\
& \times\left\{\frac{e^{-i\left\{\alpha\left(k_{j}\right) t-k_{j} x-\pi / 4\right\}}}{\alpha\left(k_{j}\right)-\sigma}-\frac{e^{-i\left\{\alpha\left(k_{j}\right) t-k_{j} x+\pi / 4\right\}}}{\alpha\left(k_{j}\right)+\sigma}\right\}
\end{aligned}
$$

where $k_{j}$ 's are finite and are real positive roots of $(4.4)$, if they exist. We write

$$
\eta=\eta_{s t}+\eta_{t r}
$$

where $\eta_{s t}$ is the steady state component while $\eta_{t r}$ is the transient component of $\eta$. The steady state component $\eta_{s t}$ comes from the contributions from the poles of the integrands $I$ and $J$ and is given by

$$
\eta_{s t} \sim \frac{e^{i\left(k_{o} x-\sigma t\right)}}{\alpha^{\prime}\left(k_{o}\right)}\left\{M E-\beta\left(k_{o}\right)\right\}
$$

This coincides with the steady state result of Rhodes Robinson [2] as $x \rightarrow \infty$ obtained by employing Green's integral theorem in the fluid region to the velocity potential and a suitable chosen Green's function assuming the time dependence to be harmonic throughout. The transient component $\eta_{t r}$ is given by (4.7).

In the absence of surface tension these results coincide with those given by Faltas [3] with suitable modifications.

\section{ASYMPTOTIC SOLUTION IN THE CASE OF INFINITE DEPTH.}

In the case of infinitely deep water, i.e., when $h \rightarrow \infty$, the functions $\beta(k)$ and $\alpha(k)$ reduce to

$$
\beta_{o}(k)=\int_{o}^{\infty} U_{1}(\xi) e^{-k \xi} d \xi, \alpha_{o}(k)=\left\{g k\left(1+M k^{2}\right)\right\}^{1 / 2}
$$

Thus the pole is the positive zero of

$$
k\left(1+M k^{2}\right)-\frac{\sigma^{2}}{g}=0
$$

and stationary points are the zeros of

$$
\frac{d \alpha_{o}}{d k}=\frac{x}{t}
$$

Now $\alpha_{o}^{\prime}(k)$ becomes positive infinity at $k=0$ and decreases sharply in the right neighbourhood of the origin. As $k$ becomes large it becomes positive infinity again. However $\alpha_{o}^{\prime}(k)$ remains positive for $k>0$. Hence there exists a global minimum of $\alpha_{o}^{\prime}(k)$ which is positive. If $x / t$ is less than this minimum value, then there is no real positive root of (5.3). However, if $x / t$ is greater than this value, then there exists almost four real positive roots.

Again we note that if $M$ is small so that its square and higher order can be neglected, then (5.2) has the real positive root given by

$$
k_{o} \simeq \frac{\sigma^{2}}{g}\left\{1-M\left(\frac{\sigma^{2}}{g}\right)^{2}\right\}
$$


and (5.3) has the real positive root given by

$$
k_{1} \simeq \frac{g t^{2}}{4 x^{2}}\left\{1+5 M\left(\frac{g t^{2}}{4 x^{2}}\right)^{2}\right\}
$$

In this case the asymptotic form of $\eta(x, t)$ can be obtained as

$$
\eta=\eta_{s t}+\eta_{t r}
$$

where

and

$$
\eta_{s t} \sim \frac{2 \sigma}{g} \frac{e^{i\left(k_{o} x-\sigma t\right)}}{\left(1+3 M k_{o}^{2}\right)}\left\{M E-\beta_{o}\left(k_{o}\right)\right\}
$$

$$
\begin{array}{r}
\eta_{t r} \sim i \sqrt{\frac{1}{2 \pi t \alpha_{o}^{\prime \prime}\left(k_{1}\right)}}\left\{\frac{M \lambda_{1}}{1+M k_{1}^{2}}-\beta_{o}\left(k_{1}\right)\right\} \\
\left\{\frac{e^{-i\left\{\alpha_{o}\left(k_{1}\right) t-k_{1} x-\pi / 4\right\}}}{\alpha_{o}\left(k_{1}\right)-\sigma}-\frac{e^{-i\left\{\alpha_{o}\left(k_{1}\right) t-k_{1} x+\pi / 4\right\}}}{\alpha_{o}\left(k_{1}\right)+\sigma}\right\}
\end{array}
$$

As before, in the absence of surface tension, these coincide with the results obtained by Faltas [3].

6. CONCLUSION.

In the presence of surface tension the steady state component $\eta_{s t}$ of the free surface depression for fluid of both finite and infinite depth given by (4.8) and (5.4) respectively, represents outgoing waves at large distances from the wavemaker and coincides with the result given by RhodesRobinson [2], while the transient component $\eta_{t r}$ exists if the global minimum of the functions $\alpha^{\prime}(k)$ or $\alpha_{o}^{\prime}(k)$ for deep fluid are less than $x / t$. However, the transient component if exists, will consist of a finite number of terms (at most four in the case of fluid of infinite depth). It may be noted that in the absence of surface tension, there exists only one positive real root of $\alpha^{\prime}(k)=x / t\left(<(g h)^{1 / 2}\right)$ or $\alpha_{o}^{\prime}(k)=x / t$ for deep fluid) and hence only one term exists for the transient component of the free surface depression in each case. However, in the presence of surface tension there may may not be any transient component of $\eta$.

Thus due to the presence of surface tension the qualitative nature of the transient component of the free surface depression changes considerably in comparison to the no surface tension case.

ACKNOWLEDGEMENT. This work is partially supported by the Third World Academy of Sciences, ICTP, Trieste, through a research scheme TWAS RG NO 274.

\section{REFERENCES}

1. HAVELOCK, T.H., Phil. Mag. 8 (1929) 569.

2. RHODES-ROBINSON, P.F., Proc. Camb. Phil. Soc. 70 (1971) 323-337.

3. FALTAS, M.S., Quart. Appl. Math. 3 (1988) 489.

4. DEBNATH, L. and ROSENBLAT, S., Quart. J. Mech. Appl. Math. 22 (1969) 221.

5. LIGHTHILL, M.J., Fourier Analysis and Generalised Functions (Camb. Univ. Press, 1962). 


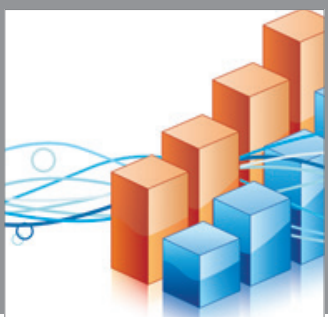

Advances in

Operations Research

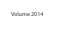

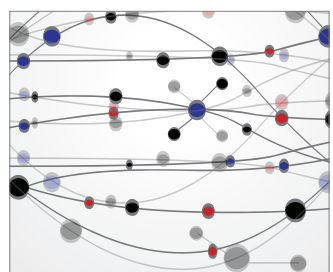

\section{The Scientific} World Journal
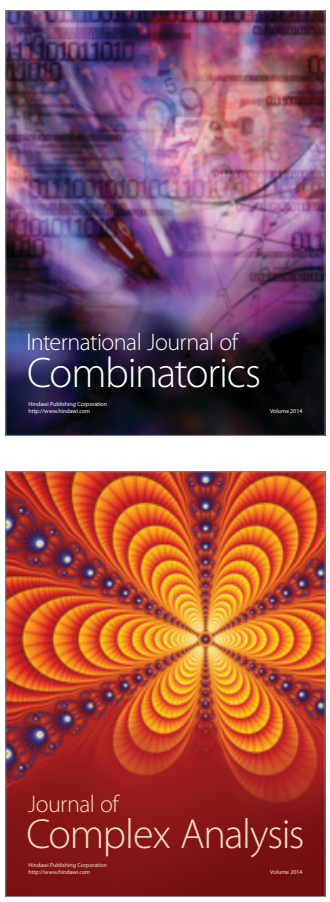

International Journal of

Mathematics and

Mathematical

Sciences
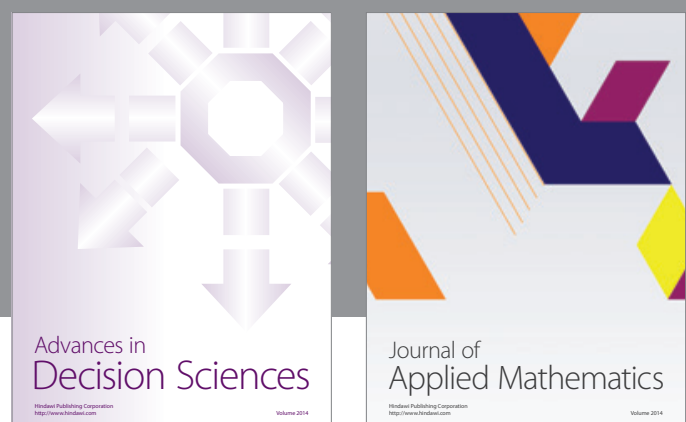

Journal of

Applied Mathematics
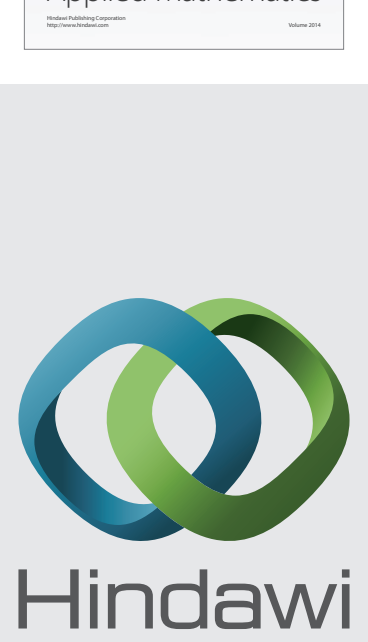

Submit your manuscripts at http://www.hindawi.com
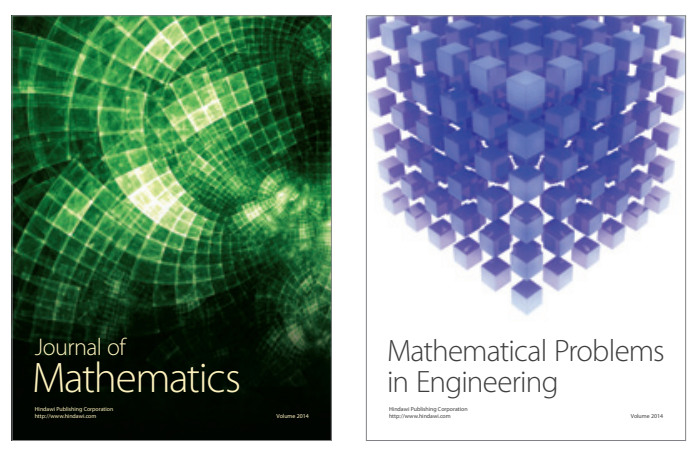

Mathematical Problems in Engineering
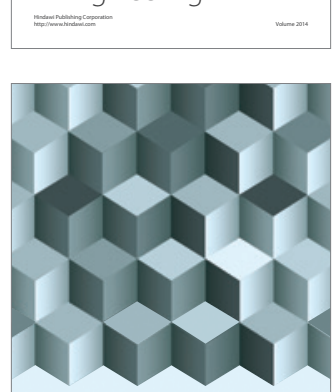

Journal of

Function Spaces
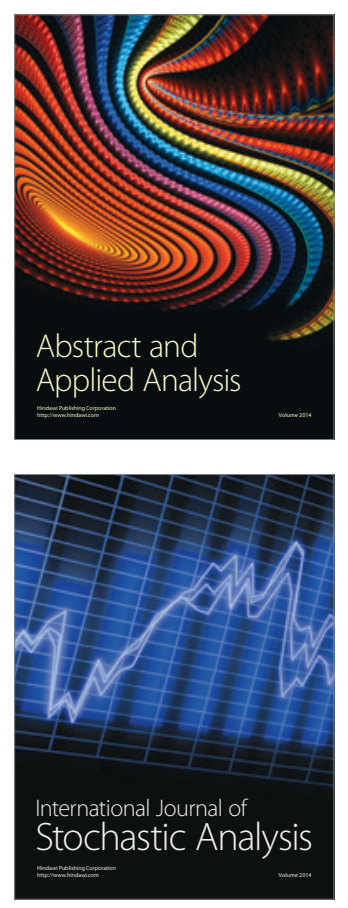

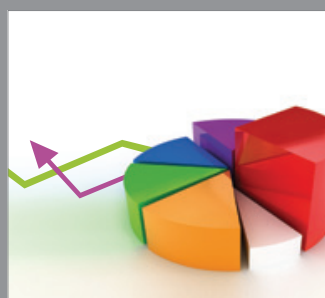

ournal of

Probability and Statistics

Promensencen
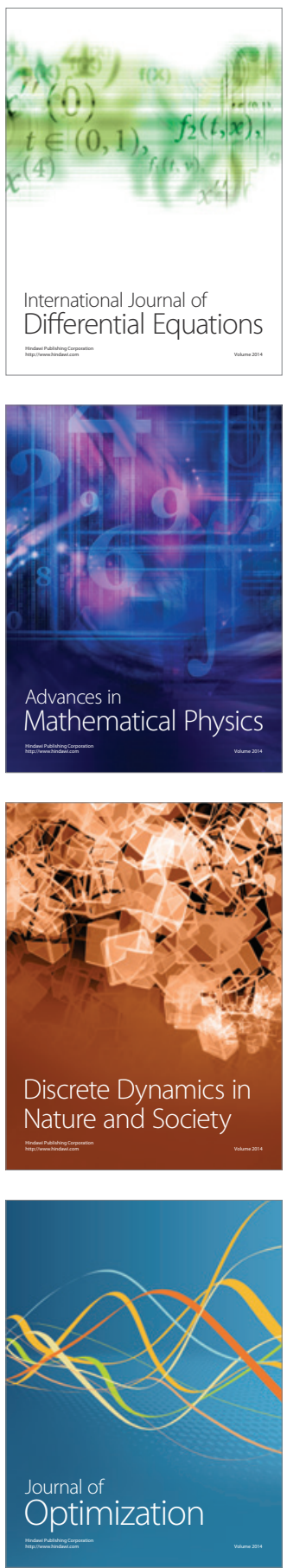